\title{
Canonical nonequilibrium statistics and applications to Fermi-Bose systems
}

\author{
W.Ebeling \\ Institute of Physics, Humboldt University, D-10115 Berlin, Germany
}

Received February 20, 2000

\begin{abstract}
The aim of this work is the study of a special class of nonequilibrium systems which admits to find exact stationary solutions of the kinetic equations. In particular we investigate canonical-dissipative systems, where the driving terms are determined by the Hamiltonian or other invariants of motion only. We construct systems which drive the system to special invariants of motion and solve the corresponding Fokker-Planck equations. Finally several applications to mean-field problems for fermion and for boson systems are discussed.
\end{abstract}

Key words: statistical mechanics, ensembles, Fokker-Planck equations, mean field, fermions, bosons

PACS: $05.40 .+j, 05.45 .+b, 05.60 .+w, 65.50 .+m$

\section{Introduction}

The development of the statistical mechanics of equilibrium systems was started in the pioneering work of Gibbs and belongs to the great achievements of natural science in the twentieth century [1-3]. The development of a corresponding statistical theory of states far from equilibrium is still far from being completed $[2,4,5]$. We will show here that there exists a special class of open systems, the so-called canonical dissipative systems, where an ensemble theory may be developed in a way which is quite similar to the Gibbs theory [6-11]. This theory is closely related to Klimontovich's statistical theory of open systems on the one hand [5] and to the theory of active Brownian particles [12-17] on the other hand. The main new topic in this work is constructing a bridge to the semiclassical kinetic theory of quantum gases proposed first by Nordheim, Uehling and Uhlenbeck $[18,19]$. Our approach is based on the theory of canonical-dissipative systems which is an extension of the statistical physics of Hamiltonian systems to a special type of dissipative systems [6-11]. The term dissipative means here that the system is non-conservative and the term canonical means that the dissipative as well as the conservative parts of the dynamics are both determined by the Hamilton function $H$ or by another invariant 
of motion. This special (and sometimes rather artificial) assumption that the dissipative terms in the dynamical equations depend only on the Hamiltonian or on certain other invariants of the motion of the system, permits us in many cases to attain exact solutions even in far-from-equilibrium situations.

\section{Dynamics of canonical-dissipative systems}

We consider a many-particle system of $f$ degrees of freedom $i=1, \ldots, f$ with the Hamiltonian $H\left(q_{1} \ldots q_{f} p_{1} \ldots p_{f}\right)$ and assume Hamiltonian equations of motion

$$
\begin{gathered}
\frac{\mathrm{d} q_{i}}{\mathrm{~d} t}=\frac{\partial H}{\partial p_{i}}, \\
\frac{\mathrm{d} p_{i}}{\mathrm{~d} t}=-\frac{\partial H}{\partial q_{i}} .
\end{gathered}
$$

Let us assume that a solution of this system is known

$$
p_{i}=p_{i}(t) ; \quad q_{i}=q_{i}(t)
$$

Geometrically seen this is a trajectory on the plane $H=E=$ const, which is defined by the initial conditions. The constant energy $E=H(t=0)$ is given by the initial conditions, which are (in certain limits) arbitrary. We construct now a canonicaldissipative system with the same Hamiltonian by means of a nondecreasing function $g(H)$ :

$$
\frac{\mathrm{d} p_{i}}{\mathrm{~d} t}=-\frac{\partial H}{\partial q_{i}}-g(H) \frac{\partial H}{\partial p_{i}}
$$

The dissipative dynamics of the so-called canonical-dissipative system $[6,10,11]$ does not conserve the energy since

$$
\frac{\mathrm{d} H}{\mathrm{~d} t}=-g(H) \sum_{i}\left(\frac{\partial H}{\partial p_{i}}\right)^{2} .
$$

A more general class of canonical-dissipative systems is obtained by introducing, beside the Hamiltonian, other invariants of motion. Let us assume that the driving functions depend on some set of other invariants of motion $I_{0}, I_{1}, I_{2}, \ldots, I_{s}$ for example

- $I_{0}=H$ - Hamilton function,

- $\mathbf{I}_{\mathbf{1}}=\mathbf{P}$ - total momentum of the system,

- $\mathbf{I}_{2}=\mathbf{L}$ - total angular momentum of the system etc. etc.

For the equation of motion we postulate

$$
\frac{\mathrm{d} p_{i}}{\mathrm{~d} t}=-\frac{\partial H}{\partial q_{i}}-\frac{\partial G\left(I_{0}, I_{1}, I_{2}, \ldots\right)}{\partial p_{i}} .
$$


In the case of equation (4) the attractor is located at the zeros $g(H)=0$, for the dynamics given by equation (6) the attractor corresponds to the maxima of the function $G\left(I_{0}, I_{1}, I_{2}, \ldots\right)$. A simple ansatz reads

$$
G\left(I_{0}, I_{1}, I_{2}, \ldots\right)=\sum_{k=0}^{f} c_{k}\left(I_{k}-I_{k}^{(0)}\right),
$$

here the $c_{k}$ are nonnegative constants and the $I_{k}^{(0)}$ denote the desired values of the invariants which correspond to the attractor.

We consider now in more detail the case $g=g(H)$. Then, in regions of the phase space where $g(H)$ is positive, the energy decays and in regions where $g(H)$ is negative, the energy increases. First we study the linear case

$$
g(H)=c\left(H-H^{(0)}\right) .
$$

Any state with $H(0)<H^{(0)}$ will increase its energy up to reaching the shell $H(t)=H^{(0)}$ and any state with $H(0)>H^{(0)}$ will decrease its energy with a rate proportional to the difference $H-H^{(0)}$. The relaxation time up to reaching the final value $H(t)=H^{(0)}$ is proportional to $c^{-1}$. The linear dissipative function (8) has found applications in the theory of dissipative Toda chains [17]. On the shell the trajectory obeys the original conservative canonical equation (2). This property has been used in the above-mentioned work to get exact solutions for canonicaldissipative Toda systems [17].

A more general dissipation function is

$$
g(H)=\gamma_{0}-\frac{(1+A) \gamma_{1}}{1+A \exp (\beta H)} .
$$

Here $A$ is a dimensionless constant, $\gamma_{0}>0$ represents the normal positive friction, $\gamma_{1}>0$ is a kind of negative friction and $\beta$ is a parameter with the meaning of a reciprocal temperature. For $\gamma_{1} \leqslant \gamma_{0}$ the friction is always positive, i.e. energy is extracted. For the opposite case $\gamma_{1}>\gamma_{0}$, we have negative friction and the system is pumped with energy at least in the same part of the phase space. Let us concentrate on this case which will allow us to drive the system to the far from equilibrium situations. In the limit $\beta \rightarrow 0$ and $A \rightarrow 0$, the dissipative function reduces to the linear case discussed above. For finite $A$ we get a friction function investigated in $[12,13,15]$.

Using the generalized canonical-dissipative dynamics defined by equation (6), the system may be driven to the maxima of the function $G\left(I_{0}, I_{1}, I_{2}, \ldots\right)$ i.e. to certain subspaces of the energy surface. For example we may fix, in addition to $H$, the total momentum or the angular momentum or other invariants. Assuming quasiergodicity we may assume that in the long run the measure of the trajectories is equally distributed on certain shells around the surfaces

$$
H\left(q_{1} \ldots q_{f} p_{1} \ldots p_{f}\right)=\mathrm{const}
$$




$$
I_{k}\left(q_{1} \ldots q_{f} p_{1} \ldots p_{f}\right)=\text { const }, \quad k=1,2, \ldots, .
$$

Assuming the ergodicity of the dynamics we may postulate microcanonical ensembles assuming that the probability density is constant on the shells

$$
I_{k}-\frac{1}{2} \delta I_{k} \leqslant I_{k}\left(q_{1} \ldots q_{f} p_{1} \ldots p_{f}\right) \leqslant I_{k}+\frac{1}{2} \delta I_{k}
$$

This means that the density is concentrated on certain shells in the phase space. The relations known from equilibrium dynamics are not valid [1]. Fluxes may be prescribed as far as they are expressed by invariants of motion. We note that this formalism is in particular well adapted to the case of integrable systems like the Toda systems, where $f$ integrals of motion are explicitely known [17].

\section{Canonical kinetic equations}

The simplest way to formulate a kinetic theory is to introduce noise into the dynamic equations which leads to Langevin equations [5].

$$
\frac{\mathrm{d} p_{i}}{\mathrm{~d} t}=-\frac{\partial H}{\partial q_{i}}-g(H) \frac{\partial H}{\partial p_{i}}+(2 D(H))^{1 / 2} \xi(t)
$$

Here $\xi(t)$ is a delta-correlated white noise. The essential assumption is, that noise and dissipation depend only on $H$. The following Fokker-Planck equation corresponds to the Langevin equation

$$
\frac{\partial \rho}{\partial t}+\sum p_{i} \frac{\partial \rho}{\partial q_{i}}-\sum \frac{\partial H}{\partial p_{i}} \frac{\partial \rho}{\partial p_{i}}=\sum \frac{\partial}{\partial p_{i}}\left[g(H) \frac{\partial H}{\partial p_{i}} \rho+D \frac{\partial \rho}{\partial p_{i}}\right] .
$$

The special structure of the dissipative and noise terms permits to find exact stationary solutions in the following form

$$
\rho_{0}\left(q_{1} \ldots q_{f} p_{1} \ldots p_{f}\right)=Q^{-1} \exp \left(-\int_{0}^{H} \mathrm{~d} H^{\prime} \frac{g\left(H^{\prime}\right)}{D\left(H^{\prime}\right)}\right) .
$$

The derivative of $\rho_{0}$ vanishes if $g(H)=0$. This means the probability is maximal at the surface $H=E_{1}$. This may be approximated by a microcanonical ensemble of the type formulated in the previous section.

For the special case of a linear dissipation function we find the stationary solution

$$
\rho_{0}\left(q_{1} \ldots q_{f} p_{1} \ldots p_{f}\right)=Q^{-1} \exp \left(\frac{c H\left(2 E_{1}-H\right)}{2 D}\right) .
$$

We mention that for these ensembles the standard equilibrium relations between energy, entropy and temperature are not valid, the mean energy and the energy dispersion being independent quantities.

The driving function given by equation (9) in combination with $D(H)=D_{0}$ leads to the distribution

$$
\rho_{0}\left(q_{1} \ldots q_{f} p_{1} \ldots p_{f}\right)=Q^{-1} \exp \left(-\frac{G_{0} g_{1}}{D_{0} d_{1}}\left[H+\left(\frac{1}{g_{1}}-\frac{1}{d_{1}}\right) \log \left(1+d_{1} H\right)\right]\right) .
$$


The same distribution follows from the combination of a linear driving

$$
g(H)=G_{0}\left(1+g_{1} H\right)
$$

and a linear noise function

$$
D(H)=D_{0}\left(1+d_{1} H\right)
$$

The existence of exact solutions for the probability distributions allows us to calculate several thermodynamic functions as the mean energy and the entropy. Further a Lyapunov-functional (the Kullback entropy) exist

$$
K\left[\rho, \rho_{0}\right]=\int \mathrm{d} q_{1} \ldots \mathrm{d} q_{f} \mathrm{~d} p_{1} \ldots \mathrm{d} p_{f} \rho \log \left[\rho / \rho_{0}\right] \geqslant 0 .
$$

This non-negative functional is always nonincreasing and the relation

$$
\frac{\mathrm{d} K\left[\rho, \rho_{0}\right]}{\mathrm{d} t} \leqslant 0
$$

describes a monotonic relaxation to the stationary state. This unequality guaranteess the uniqueness of the stationary solution.

\section{Mean-field fermion-boson systems}

The only case of canonical-dissipative systems which was studied in detail so far is the theory of active Brownian particles without interactions [13,16]. The theory reduces then to a one-particle problem since $P(1,2, \ldots N)=\Pi P(i)$. We assume for the one-particle Hamiltonian (with $m=1$ )

$$
H_{i}=p_{i}^{2} / 2
$$

Therefore all noninteracting systems with $g=g\left(p^{2}\right)$ are of canonical-dissipative type. We mention that Fokker-Planck equations for such systems with nonlinear friction have already been discussed in detail by Klimontovich [5].

In our earlier work we studied the special dissipative function closely related to equation (9)

$$
g(H)=\gamma_{0}-\frac{q d}{c+2 d H} .
$$

This model describes the driven particles which possess an energy depot. Assuming for the noise an Einstein relation $D=k T \gamma_{0}$, the distribution functions and several kinetic characteristics such as the mean square displacement were calculated [16]. Further several results for the one-particle dynamics in parabolic fields were obtained $[13,16]$. Instead of an external field we introduce here a mean field generated by the particles themselves. 
Let us consider physical particles in a 3d-space with the coordinates $\vec{r}$ and momenta $\vec{p}=m \dot{\vec{r}}$ and assume that the one-particle dynamics is generated by the mean-field Hamiltonian

$$
H_{1}=\frac{p^{2}}{2 m}+U(\vec{r})
$$

where $U$ is the self-consistent Vlasov field

$$
U(\vec{r})=V_{\text {ext }}(\vec{r})+\int \mathrm{d} \vec{r} V\left(\vec{r}-\vec{r}^{\prime}\right) \int \mathrm{d} \vec{p} f(\vec{p}, \vec{r}) .
$$

Here the interaction potential should be integrable such as the Coulomb field. We generate now a one-particle dynamics, corresponding to this mean-field Hamiltonian by

$$
\frac{\mathrm{d} \vec{p}}{\mathrm{~d} t}=-\frac{\partial U}{\partial \vec{r}}-g\left(H_{1}\right) \vec{p}+(2 D)^{1 / 2} \vec{\xi}(t)
$$

with the dissipative functions

$$
g\left(H_{1}\right)=\gamma_{0}-\frac{(1+A) \gamma_{1}}{1+A \exp \left(\beta H_{1}\right)}
$$

We will show that this system generates a nonequilibrium Thomas-Fermi-type dynamics. For that instance we begin with a special choice of the parameters namely

$$
\begin{aligned}
& (1+A) \gamma_{1}=\gamma_{0}, \\
& A=\exp (-\beta \mu), \\
& D=D_{0}=\frac{\gamma_{0}}{m \beta} .
\end{aligned}
$$

Here $\beta$ is the reciprocal temperature of the physical system and $\mu$ the chemical potential. The friction constant $\gamma_{0}$ is then the only remaining nonequilibrium constant which determines the time of relaxation to the stationary state; in our phenomenological approach $\gamma_{0}$ is a free constant which might be adapted to the physical system we would like to study. For Coulomb systems a reasonable choice for the effective friction may be the Landau approximation [5]

$$
\gamma_{0}=\text { const } \cdot \frac{n e^{4}}{\left(k_{\mathrm{B}} T\right)^{3 / 2}} \log \frac{k_{\max }}{k_{\min }},
$$

where $e$ is the charge and $k_{\max }, k_{\min }$ are maximal/minimal parameters of the scattering process.

We note that our system is not an active system since $g\left(H_{1}\right)$ is always nonnegative. In other words the special mean-field system we have defined is not a nonequilibrium system, its stationary solution should correspond to equilibrium situations. At the first glance the system defined this way is not very physical. However, it has a very interesting property of its stationary solution relating to the 
Thomas-Fermi distribution. Indeed the Fokker-Planck equation corresponding to equation (26) which reads

$$
\frac{\partial \rho(\vec{r}, \vec{p})}{\partial t}+\vec{p} \nabla_{r} \rho-\nabla_{r} U \nabla_{p} \rho=\nabla_{p}\left[\vec{p} g(H) \rho+D_{0} \nabla_{p} \rho\right]
$$

possesses the stationary solution

$$
\rho(\vec{r}, \vec{p})=f_{0}\left(H_{1}\right)=\frac{1}{1+A \exp \left(\beta p^{2} / 2 m+\beta U(\vec{r})\right)} .
$$

Substituting here equation (29) we find the Thomas-Fermi distribution with $\mu$ being the chemical potential. Rewriting equation (27) in the form

$$
g\left(H_{1}\right)=\gamma_{0}\left(1-f_{0}\left(H_{1}\right)\right)
$$

we clearly see the physical reason for the realization of the Thomas-Fermi distribution: In the parts of the phase which are unoccupied $f_{0}=0$ the particles feel a normal friction $\gamma_{0}$ whereas particles in the occupied parts $f_{0} \simeq 1$ feel an additional acceleration which removes them from the occupied parts of the phase space.

We note that the choice proposed above is not unique, all combinations $\frac{g(H)}{D(H)}$ with the property

$$
\frac{g\left(H_{1}\right)}{D\left(H_{1}\right)}=\frac{1}{k_{\mathrm{B}} T}\left(1-f_{0}\left(H_{1}\right)\right)
$$

lead to Thomas-Fermi distributions.

The method may be easily transferred to the dynamics of particles with Bose statistics. Using the following value for the constant $A$ :

$$
A=-\exp (-\beta \mu)
$$

we get the friction function

$$
g\left(H_{1}\right)=\gamma_{0}\left(1-\frac{1}{1-\exp \left(\beta H_{1}-\beta \mu\right)}\right) .
$$

Then our Fokker-Planck equation (32) possesses the exact stationary solution

$$
\rho(\vec{r}, \vec{p})=f_{0}\left(H_{1}\right)=\frac{1}{\exp \left(\beta p^{2} / 2 m+\beta U(\vec{r})-\beta \mu\right)-1},
$$

which is a Bose distribution.

We do not claim here that this simple model of fermionic or bosonic dynamics gives the correct kinetic properties but it provides at least a relaxation to the correct thermodynamics and may therefore be used like a Monte-Carlo method. We mention that our method is in the same spirit as the ad hoc ansatz for kinetic equations of quantum particles which was proposed around 70 years ago by Nordheim, Uehling and Uhlenbeck which is still used in many recent applications [20,21].

It may be worth mentioning that the realization of the symmetry effects in many particle simulations is by far not a trivial task [22].

The range of applicability of the new method presented here however remains open and needs further detailed investigations and numerical experiments. 


\section{Discussion}

This work is devoted to the study of canonical-dissipative systems which include dissipative effects in a rather special form. Our main aim was

(i) to find exact solutions for model equations, with an arbitrarily prescribed energy;

(ii) to discuss several variants of dissipative effects including active friction;

(iii) to derive explicite solutions for the stationary distribution functions;

(iv) to discuss possible applications to mean-field quantum gases.

We started our work from the Hamiltonian theory for conservative mechanical systems. In order to extend the known solutions for conservative systems to nonconservative systems we used the general theory of canonical-dissipative systems. Special canonical-dissipative systems were constructed which solution converges to the solution of the conservative system with the given energy or other given invariants of motion. In this way we are able to generate states with prescribed energy. Given any initial condition, the system we have constructed will converge to the solution of the Hamilton equations on an energy surface which we can give in advance.

Further, we constructed and analyzed several special ways of dissipative effects. Among the possible applications we stressed here in particular a new way to model the Pauli principle in a semiclassical way by means of special dissipative effects.

There is no space here to explain in more detail other interdisciplinary applications of the theory of open systems with supply of free energy. Let us give just the references to three interesting trends related to modelling the biological mobility $[12,23,24]$.

The author would like to thank R.Graham, J.Ortner, Y.Pomeau and H.H.Wolter for discussions.

\section{References}

1. Gibbs J.W. Elementary Principles in Statistical Mechanics. 1902. - Reprinted in Collected Works of J. Willard Gibbs, New Haven, Yale Univ. Press, 1931.

2. Bogoliubov N.N. Problems of Dynamical Theory in Statistical Physics. Moscow, Gostekhisdat, 1946 (in Russian).

3. Yukhnovsky I.R., Holovko M.F. Statistical Mechanics of Classical Equilibrium Systems. Kiev, Naukova Dumka, 1980 (in Russian).

4. Zubarev D.N., Röpke G., Morozov A.A. Statistical Mechanics of Nonequilibrium Processes. vol. I, II. Weinheim - Berlin, VCH Wiley, 1996, 1997.

5. Klimontovich Yu.L. Theory of Open systems. Dordrecht, Kluwer, 1995.

6. Graham R. // Springer Tracts in Mod. Phys., 1973, vol. 66, p. 111-150.

7. Haken H. // Z. Phys., 1973, vol. 263, p. 267-275.

8. Hongler M.O., Ryter D.M. // Z. Phys. B, 1978, vol. 31, p. 333-343.

9. Ebeling W., Engel-Herbert H. // Physica A, 1989, vol. 104, p. 378-390.

10. Ebeling W. // Springer Series in Synergetics (Ed. Haken H., Berlin, Springer), 1981, vol. 11, p. 188-199.

11. Feistel R., Ebeling W. Evolution of Complex Systems. Dordrecht, Kluwer, 1989.

12. Schweitzer F., Ebeling W., Tilch B. Complex motion of brownian particles with energy depots. // Phys. Rev. Lett., 1998, vol. 80, p. 5044-5047. 
13. Ebeling W., Schweitzer F., Tilch B. Active brownian particles with energy depots modelling animal mobility. // BioSystems, 1999, vol. 49, p. 17-29.

14. Schweitzer F., Tilch B., Ebeling W. Uphill motion of active brownian particles in piecewise linear potentials. // Europ. Phys. J. B, 2000, vol. 14, p. 157-168.

15. Tilch B., Schweitzer F., Ebeling W. Directed motion of brownian particles with internal energy depot. // Physica A, 1999, vol. 273, p. 294-314.

16. Erdmann U., Ebeling W., Schimansky-Geier L., Schweitzer F. Brownian particles far from equilibrium. // Europ. Phys. J. B, 2000, vol. 15, p. 105-113.

17. Makarov V., Ebeling W., Velarde M. // Int. J. Bifurc. \& Chaos, 2000, vol. 10, No. 5 (in press).

18. Nordheim L.W. // Proc. Roy. Soc. London A, 1928, vol. 119, p. 689-699.

19. Uehling E.A., Uhlenbeck G.E. // Rhys. Rev., 1932, vol. 43, p. 552-562.

20. Lacaze R., Lallemand P., Pomeau Y., Rica S. Dynamical formation of a Bose-Einstein condensate. Preprint Orsay/Paris, 1999; Pomeau Y., Brachet M.-E., Metens S., Rica S. Theorie cinetique d'un gaz de Bose dilue avec condensat. Preprint Paris/Madrid, 1999 (in French).

21. Wolter H.H. Kinetic equations for relativistic heavy ion collisions. - In: Lecture Notes, Rostock/Prerow, 1999.

22. Ebeling W., Schautz F. // Phys. Rev. E, 1997, vol. 56, p. 3498-3507.

23. Vicsek T., Czirok A., Ben-Jacob E., Cohen I., Shochet O. Novel type of phase transition in a system of self-driven particles. // Phys. Rev. Lett., 1995, vol. 75, p. 1226-1229.

24. Bier M., Astumian R.D. // Phys. Rev. Lett., 1996, vol. 76, p. 4277-4285.

\title{
Канонічна нерівноважна статистика і застосування до Фермі-Бозе систем
}

\author{
У.Ебелінґ \\ Інститут фізики, університет Гумбольдта, \\ D-10115 Берлін, Німеччина \\ Отримано 20 лютого 2000 р.
}

Метою цієї роботи є вивчення особливого класу нерівноважних систем, який допускає знаходження стаціонарних розв'язків кінетичних рівнянь. Зокрема, ми досліджуємо канонічно-дисипативні системи, в яких ведучі члени визначаються гамільтоніаном або іншими інваріантами руху. Ми будуємо системи, які приводять систему до особливих інваріантів руху, і розв'язуємо відповідні рівняння ФокераПланка. Нарешті, ми обговорюємо деякі застосування до середньопольових проблем для систем ферміонів і бозонів.

Ключові слова: статистична механіка, ансамблі, рівняння Фокера-Планка, середнє поле, ферміони, бозони

PACS: $05.40 .+j, 05.45 .+b, 05.60 .+w, 65.50 .+m$ 
